K. TOHGE

KODAI MATH. J.

16 (1993). 379-397

\title{
THE LOGARITHMIC DERIVATIVE AND A HOMOGENEOUS DIFFERENTIAL POLYNOMIAL OF A MEROMORPHIC FUNCTION
}

\author{
By KazUya TOHge
}

\section{Introduction}

In this note, by a meromorphic function we mean a function meromorphic in the complex plane $\boldsymbol{C}$. We shall here assume that the reader is familiar with the standard notation and terminology of value distribution theory (see for example, Hayman [1] or [3]). For a meromorphic function $g(z)$, which does not vanish identically, we can consider the logarithmic derivative $g^{\prime}(z) / g(z)$. It plays an important role in Nevanlinna's theory of meromorphic functions. The following occupies the main part.

LEMMA. Let $g(z)$ be a meromorphic function. If $g(z)$ is transcendental, we have

$$
m\left(r, \frac{g^{\prime}}{g}\right)=O\left(\log ^{+} T(r, g)+\log r\right)
$$

as $r \rightarrow \infty$ through all values if $g(z)$ has finite order and as $r \rightarrow \infty$ outside a set of $r$ of finite linear measure otherwise. If $g(z)$ is a rational function and not identically equal to zero,

$$
m\left(r, \frac{g^{\prime}}{g}\right)=o(1)
$$

as $r \rightarrow \infty$ through all values.

For the sake of simplicity, we shall use the symbol "n.e. (nearly everywhere)" instead of tediously saying that possibly outside a set of $r$ of finite linear measure.

W. K. Hayman pointed out the necessity of treating a homogeneous differential polynomial $g^{\prime \prime} g-2 g^{\prime 2}$ of an entire function $g(z)$ in his famous book $[1$; $\S 3.6$, p. 77]. Concerning this proposal E. Mues [4] studied an influence of the zeros of $g^{\prime \prime} g-a g^{\prime 2}$ with a complex number $a$ on the entire function $g(z)$ itself.

Received April 28, 1992. 
He proved that if $g^{\prime \prime} g-a g^{\prime 2}$ has no zero, then $g(z)=\exp (\alpha z+\beta)$ are the only transcendental functions with this property if $a \neq 1$. This result settled a question of Hayman made in [1]. Our purpose of this note is to give an estimate of the zeros of $g(z)$ by those of the homogeneous differential polynomial $g^{\prime \prime} g-$ $a g^{\prime 2}$. With the equation

$$
T\left(r, \frac{g^{\prime}}{g}\right)=m\left(r, \frac{g^{\prime}}{g}\right)+\bar{N}(r, 0, g)+\bar{N}(r, g)
$$

for a meromorphic function $g(z) \neq 0$ and the above lemma, it gains our purpose to estimate the characteristic function of a logarithmic derivative by the counting function with respect to the zeros of a homogeneous differential polynomial. Our method to obtain such a result is based on arriving at a homogeneous linear equation in $g^{\prime}$ and $g$ after a linearization of $g^{\prime \prime} g-a g^{\prime 2}$. The term "linearization" was introduced by M. Ozawa [5], and Mues [4], Ozawa, G. Frank and others have made frequent use of this method. We now represent the differential polynomial $g^{\prime \prime} g-a g^{\prime 2}$ by means of a Wronskian determinant

$$
W\left(f_{1}, f_{2}\right)=f_{1} f_{2}{ }^{\prime}-f_{1}{ }^{\prime} f_{2} .
$$

We have indeed for a constant $a \equiv C$

$$
W\left((a-1) z g^{\prime}(z)+g(z), g^{\prime}(z)\right)=g^{\prime \prime}(z) g(z)-a g^{\prime}(z)^{2},
$$

which we denote by $W_{a}(z)$. That is a reason why we can treat this homogeneous differential polynomial $W_{a}(z)$.

We shall naturally consider only the case where $W_{a}(z)$ does not vanish identically. Because if $W_{a}(z) \equiv 0$, two functions $(a-1) z g^{\prime}(z)+g(z)$ and $g^{\prime}(z)$ are linearly dependent over $C$. Then there exist two constants $C_{1}$ and $C_{2}$, at least one of which is different from zero, such that an equation

$$
\left(C_{1}(a-1) z+C_{2}\right) g^{\prime}(z)+C_{1} g(z)=0
$$

holds. If $C_{1}(a-1) z+C_{2} \equiv 0$, we have $C_{2}=0$ and $\alpha=1$. By (1.4) it thus follows $g(z) \equiv 0$, which is a contradiction. Hence unless $g(z) \equiv 0$, it is equal to $\exp \left(-C_{1} z / C_{2}+\right.$ const.) if $a=1$ (and thus $\left.C_{2} \neq 0\right)$, and $g(z)=C_{3}\left(C_{1}(a-1) z+C_{2}\right)^{-1 /(a-1)}$, $C_{3} \in-C-\{0\}$, if $a \neq 1$. If $C_{1} \neq 0$ in the latter case, the exponent $-1 /(a-1)$ must be an integer $m(\neq 0)$, say. The following is a summary of this trivial observation :

The meromorphic functions $g(z)$ with the property $W_{a}(z) \equiv 0$ are reduced to the next three: for $\alpha(\neq 0), \beta \in C$,

$1^{\circ} . g(z) \equiv \beta$, when $a$ is any complex number;

$2^{\circ}$. $g(z)=\exp (\alpha z+\beta)$, when $a=1$;

$3^{\circ}$. $g(z)=(\alpha z+\beta)^{m}$, when $a=(m-1) / m$ with a non-zero integer $m$. 


\section{Results}

We shall prove the following theorem which gives a desired estimate of the logarithmic derivative $g^{\prime}(z) / g(z)$.

THEOREM. Let $g(z)$ be a non-constant meromorphic function and define a homogeneous differential polynomial $W_{a}(z)$ in $g(z)$ for a complex number $a$ by (1.3). If $W_{a}(z)$ does not vanish identically, then an inequality

$$
T\left(r, \frac{g^{\prime}}{g}\right) \leqq A_{a} m\left(r, \frac{g^{\prime}}{g}\right)+B_{a} m\left(r, \frac{W_{a}^{\prime}}{W_{a}}\right)+C_{a}\left\{\bar{N}\left(r, 0, W_{a}\right)+\bar{N}(r, g)\right\}+U_{a}(r)
$$

holds as $r \rightarrow \infty$, except for two cases (i), (ii) below. Here the constants $A_{a}, B_{a}, C_{a}$ depend only on the number $a$ and satisfy

$$
0 \leqq A_{a} \leqq\left\{\begin{array}{ll}
4, & \text { if } a \neq 1,1 / 2, \\
2, & \text { if } a=1, \\
1, & \text { if } a=1 / 2,
\end{array} \quad 0 \leqq B_{a} \leqq\left\{\begin{array}{ll}
5, & \text { if } a \neq 1,1 / 2,0, \\
2, & \text { if } a=1, \\
4, & \text { if } a=1 / 2, \\
1, & \text { if } a=0,
\end{array} \quad 0 \leqq C_{a} \leqq 5\right.\right.
$$

for any $a$, and also $U_{a}(r)$ is a real-valued function on $[0, \infty)$ such that if we fix the number $a$, then it satisfies

$$
U_{a}(r)=\left\{\begin{array}{l}
O\left[\log ^{+} T\left(r, \frac{g^{\prime}}{g}\right)+\log ^{+} m\left(r, \frac{W_{a}{ }^{\prime}}{W_{a}}\right)+\log ^{+}\left\{\bar{N}\left(r, 0, W_{a}\right)+\bar{N}(r, g)\right\}+\log r\right], \\
\quad \text { if } a \pm 1 / 2,0, \\
O\left[\log ^{+} T\left(r, \frac{g^{\prime}}{g}\right)+\log ^{+}\left\{\bar{N}\left(r, 0, W_{0}\right)+\bar{N}(r, g)\right\}+\log r\right], \quad \text { if } a=0, \\
O(1), \quad \text { if } a=1 / 2,
\end{array}\right.
$$

as $r \rightarrow \infty$ possibly outside a set $E_{a}$ of $r$ of finite linear measure depending on the number $a$.

(i) When $a=1 / 2, g(z)=\alpha z^{2}+\beta z+\gamma$, where $\alpha, \beta$, $\gamma$ are complex constants with $\beta^{2}-4 \alpha \gamma \neq 0$; and

(ii) when $a=1, g(z)=C_{1} e^{\lambda_{1} z}+C_{2} e^{\lambda_{2} z}$, where $\lambda_{1}, \lambda_{2}, C_{1}, C_{2}$ are complex constants with $\lambda_{1} \neq \lambda_{2}$ and $C_{1} \cdot C_{2} \neq 0$;

are the exceptions as mentioned above.

Remark. It is easy to see that $g(z)$ as in the cases (i) and (ii) indeed fails to satisfy the inequality (2.1). In fact:

(i). $\quad g(z)=\alpha z^{2}+\beta z+\gamma$ gives $W_{1 / 2}(z) \equiv-(1 / 2)\left(\beta^{2}-4 \alpha \gamma\right)(\neq 0)$. Then we deduce

and

$$
\begin{aligned}
& m\left(r, g^{\prime} / g\right)=o(1), \\
& m\left(r, W_{1 / 2}^{\prime} / W_{1 / 2}\right)=\bar{N}\left(r, 0, W_{1 / 2}\right)=\bar{N}(r, g) \equiv 0,
\end{aligned}
$$

$$
U_{1 / 2}(r)=O(1)
$$


as $r \rightarrow \infty$, while

$$
T\left(r, g^{\prime} / g\right)=m\left(r, g^{\prime} / g\right)+N\left(r, g^{\prime} / g\right)=\varepsilon \log r+o(1)
$$

as $r \rightarrow \infty$, with $\varepsilon=1$ if $\alpha=0$ and $\varepsilon=2$ if $\alpha \neq 0$.

(ii). In this case, $W_{1}(z)=\left(\lambda_{1}-\lambda_{2}\right)^{2} C_{1} C_{2} e^{\left(\lambda_{1}+\lambda_{2}\right) z}$, and that $g(z)$ is an entire function of order 1 . Thus we have

and

$$
\begin{aligned}
& m\left(r, g^{\prime} / g\right)=O(\log r), \\
& m\left(r, W_{1}^{\prime} / W_{1}\right)=O(\log r), \\
& \bar{N}\left(r, 0, W_{1}\right)=\bar{N}(r, g) \equiv 0,
\end{aligned}
$$

$$
U_{1}(r)=O(\log r) \quad \text { as } r \rightarrow \infty .
$$

Using an expression $g(z)=C_{1} e^{\lambda_{2} z}\left\{e^{\left(\lambda_{1}-\lambda_{2}\right) z}+C_{2} / C_{1}\right\}$, we are led to

$$
T\left(r, g^{\prime} / g\right)=\bar{N}(r, 0, g)+O(\log r)=\frac{\left|\lambda_{1}-\lambda_{2}\right|}{\pi} r+O(\log r)
$$

as $r \rightarrow \infty$ (see for example, Hayman [1: p. 7]), however.

Our way to prove this theorem also applies to the following

COROLLARY. Besides the hypothesis of our theorem we assume that $g(z)$ is an entire function and that as $r \rightarrow \infty$, n.e.,

$$
m\left(r, W_{a}\right)=o\{m(r, g)\} .
$$

Then $W_{a}(z)$ must be a constant $(\neq 0)$ and $g(z)$ is at least one of the following;

(i) when $a=1 / 2, g(z)=\alpha z^{2}+\beta z+\gamma$, where $\alpha, \beta, \gamma=C$ with $\beta^{2}-4 \alpha \gamma \neq 0$;

(ii) when $a=1, g(z)=C_{1} e^{\lambda z}+C_{2} e^{-\lambda z}$, where $\lambda, C_{1}, C_{2} \equiv C-\{0\}$; and

(iii) when $a \neq 0,1 / 2, g(z)=\alpha z+\beta$, where $\alpha(\neq 0), \beta \leftrightharpoons C$.

Remarks $1^{\circ}$. If we further suppose that $g(z)$ is of finite order $\rho$ and $W_{a}(z)$ has the order $\lambda$ satisfying $\lambda<\rho$, the case (ii) is the only possible one and then $\rho=1$ and $\lambda=0$ (in particular, $W_{1}(z)$ is a constant). We may regard it as a partial answer to a problem of A. Edrei (see Hayman [2: Problem 2.25]) when $f=g^{\prime}$ there.

$2^{\circ}$. Replacing the condition (2.2) by

$$
T\left(r, W_{a}\right)=o\{T(r, g)\} \quad \text { as } r \rightarrow \infty, \text { n. e., }
$$

we can prove this result for meromorphic functions with the property such that

$$
N(r, g)=o\{T(r, g)\} \quad \text { as } r \rightarrow \infty \text {, n. e.. }
$$




\section{Proof of Theorem: A Preparation}

Because of $W_{a}(z) \not \equiv 0$, we can consider a second order linear ordinary differential equation

$$
w^{\prime \prime}+G_{a}(z) w^{\prime}+H_{a}(z) w=0
$$

with the coefficients

$$
G_{a}(z)=-\frac{W_{a}^{\prime}(z)}{W_{a}(z)}
$$

and

$$
H_{a}(z)=\frac{W\left(\left\{(a-1) z g^{\prime}(z)+g(z)\right\}^{\prime}, g^{\prime \prime}(z)\right)}{W_{a}(z)} .
$$

Since (3.1) is written as $\left(W\left[w,(a-1) z g^{\prime}+g, g^{\prime}\right] / W_{a}\right)=0$, two functions $(a-1) z g^{\prime}(z)+g(z)$ and $g^{\prime}(z)$ form a fundamental system of this equation. Firstly for $w=(a-1) z g^{\prime}(z)+g(z)$ Equation (3.1) gives

$$
\begin{aligned}
& (a-1) z\left\{g^{\prime \prime \prime}(z)+G_{a}(z) g^{\prime \prime}(z)+H_{a}(z) g^{\prime}(z)\right\} \\
& \quad+(2 a-1) g^{\prime \prime}(z)+a G_{a}(z) g^{\prime}(z)+H_{a}(z) g(z)=0 .
\end{aligned}
$$

Also for $w=g^{\prime}(z)$,

$$
g^{\prime \prime \prime}(z)+G_{a}(z) g^{\prime \prime}(z)+H_{a}(z) g^{\prime}(z)=0 .
$$

Together with (3.4) the first equation is reduced to

$$
(2 a-1) g^{\prime \prime}(z)+a G_{a}(z) g^{\prime}(z)+H_{a}(z) g(z)=0 .
$$

The two, (3.4) and (3.5), are called a linearization of the differential polynomial $W_{a}(z)$. Eliminating $g^{\prime \prime \prime}(z)$ and $g^{\prime \prime}(z)$ from them we shall obtain an equation in $g^{\prime}(z)$ and $g(z)$. To do this, we differentiate both sides of (3.5) with respect to $z$ and get

$$
(2 a-1) g^{\prime \prime \prime}(z)+a G_{a}(z) g^{\prime \prime}(z)+\left(a G_{a}{ }^{\prime}(z)+H_{a}(z)\right) g^{\prime}(z)+H_{a}{ }^{\prime}(z) g(z)=0 .
$$

Using (3.5) and (3.6) we reduce Equation (3.4) to

$$
\begin{aligned}
& \left\{a(2 a-1) G_{a}{ }^{\prime}(z)+a(a-1) G_{a}(z)^{2}-2(a-1)(2 a-1) H_{a}(z)\right\} g^{\prime}(z) \\
& \quad=-\left\{(2 a-1) H_{a}{ }^{\prime}(z)+(a-1) G_{a}(z) H_{a}(z)\right\} g(z),
\end{aligned}
$$

which is the homogeneous linear equation in $g^{\prime}(z)$ and $g(z)$ as desired. For the sake of simplicity, we denote the coefficients by $\phi_{a}(z)$ and $\phi_{a}(z)$, i. e.,

$$
\phi_{a}(z):=a(2 a-1) G_{a}{ }^{\prime}(z)+a(a-1) G_{a}(z)^{2}-2(a-1)(2 a-1) H_{a}(z),
$$




$$
\psi_{a}(z):=-(2 a-1) H_{a}{ }^{\prime}(z)-(a-1) G_{a}(z) H_{a}(z) .
$$

Then the above equation (3.7) is of the form

$$
\phi_{a} \cdot g^{\prime}=\phi_{a} \cdot g \text {. }
$$

Now it makes all the difference in methods whether or not $\phi_{a} \equiv 0$.

\section{Proof of Theorem: Case I where $\phi_{a} \not \equiv 0$}

Since $g \neq 0$, Equation (3.10) gives an expression of the logarithmic derivative, i. e.,

$$
\frac{g^{\prime}}{g}=\frac{\psi_{a}}{\phi_{a}}
$$

We shall now distinguish the cases about the value of $a$ to study the value distribution of meromorphic functions $G_{a}, H_{a}, \phi_{a}$, and $\phi_{a}$.

Subcase i. $a$ is different from $0,1 / 2,1$.

Using Equation (3.5) we have

$$
\begin{aligned}
H_{a} & =-(2 a-1) \frac{g^{\prime \prime}}{g}-a G_{a} \frac{g^{\prime}}{g} \\
& =-(2 a-1)\left\{\left(\frac{g^{\prime}}{g}\right)^{\prime}+\left(\frac{g^{\prime}}{g}\right)^{2}\right\}-a G_{a} \frac{g^{\prime}}{g} .
\end{aligned}
$$

Thus we apply Lemma to (4.2) and obtain

$$
m\left(r, G_{a}\right)=m\left(r, \frac{W_{a}^{\prime}}{W_{a}}\right)
$$

and

$$
\begin{aligned}
m\left(r, H_{a}\right) & =m\left(r, \frac{g^{\prime}}{g}\left\{-(2 a-1) \frac{\left(g^{\prime} / g\right)^{\prime}}{\left(g^{\prime} / g\right)}-(2 a-1) \frac{g^{\prime}}{g}-a G_{a}\right\}\right) \\
& \leqq 2 m\left(r, \frac{g^{\prime}}{g}\right)+m\left(r, G_{a}\right)+m\left(r, \frac{\left(g^{\prime} / g\right)^{\prime}}{\left(g^{\prime} / g\right)}\right)+O(1) \\
& \leqq 2 m\left(r, \frac{g^{\prime}}{g}\right)+m\left(r, \frac{W_{a}^{\prime}}{W_{a}}\right)+O\left\{\log ^{+} T\left(r, \frac{g^{\prime}}{g}\right)+\log r\right\},
\end{aligned}
$$

as $r \rightarrow \infty$, n.e.. Since the poles of $W_{a}$ occur possibly at those of $g$, we have

$$
\bar{N}\left(r, W_{a}\right) \leqq \bar{N}(r, g),
$$

and thus

$$
\begin{aligned}
N\left(r, G_{a}\right) & =N\left(r, \frac{W_{a}^{\prime}}{W_{a}}\right)=\bar{N}\left(r, W_{a}\right)+\bar{N}\left(r, 0, W_{a}\right) \\
& \leqq \bar{N}\left(r, 0, W_{a}\right)+\bar{N}(r, g) .
\end{aligned}
$$


Whenever $H_{a}$ as well as $G_{a}$ has a pole, $W_{a}$ has a zero or $g$ has a pole. Its multiplicity is at most two as we see from (4.2). Thus

$$
N\left(r, H_{a}\right) \leqq 2\left\{\bar{N}\left(r, 0, W_{a}\right)+\bar{N}(r, g)\right\} .
$$

If $G_{a}$ does not vanish identically, an application of Lemma to (3.8) implies

$$
\begin{aligned}
m\left(r, \phi_{a}\right) & \leqq m\left(r, a G_{a}\left\{(2 a-1) \frac{G_{a}^{\prime}}{G_{a}}+(a-1) G_{a}\right\}\right)+m\left(r, H_{a}\right)+O(1) \\
& \leqq 2 m\left(r, G_{a}\right)+m\left(r, \frac{G_{a}{ }^{\prime}}{G_{a}}\right)+m\left(r, H_{a}\right)+O(1) \\
& \leqq 2 m\left(r, G_{a}\right)+m\left(r, H_{a}\right)+O\left\{\log ^{+} T\left(r, G_{a}\right)+\log r\right\}
\end{aligned}
$$

as $r \rightarrow \infty$, n. e.. This is also valid when $G_{a} \equiv 0$ so that $\phi_{a}=-2(a-1)(2 a-1) H_{a}$. We see that $H_{a}$ is not constantly equal to zero. In fact otherwise, $\phi_{a} \equiv 0$ by (3.9) and therefore $g^{\prime}(z) \equiv 0$ by (4.1), which is a contradiction. It follows from (3.9)

$$
\begin{aligned}
m\left(r, \psi_{a}\right) & =m\left(r,-H_{a}\left\{(2 a-1) \frac{H_{a}^{\prime}}{H_{a}}+(a-1) G_{a}\right\}\right) \\
& \leqq m\left(r, H_{a}\right)+m\left(r, G_{a}\right)+O\left\{\log ^{+} T\left(r, H_{a}\right)+\log r\right\}
\end{aligned}
$$

as $r \rightarrow \infty$, n.e.. We can observe the poles of $\phi_{a}$ and $\phi_{a}$ similarly to those of $G_{a}$ and $H_{a}$ as in (4.5) and (4.6), respectively, i. e.,

$$
N\left(r, \phi_{a}\right) \leqq 2\left\{\bar{N}\left(r, 0, W_{a}\right)+\bar{N}(r, g)\right\},
$$

and

$$
N\left(r, \psi_{a}\right) \leqq 3\left\{\bar{N}\left(r, 0, W_{a}\right)+\bar{N}(r, g)\right\} .
$$

From the estimates (4.7), (4.8), (4.9) and (4.10) we arrive at

and

$$
\begin{aligned}
T\left(r, \phi_{a}\right) \leqq & 2 m\left(r, G_{a}\right)+m\left(r, H_{a}\right)+2\left\{\bar{N}\left(r, 0, W_{a}\right)+\bar{N}(r, g)\right\} \\
& +O\left\{\log ^{+} T\left(r, G_{a}\right)+\log r\right\}
\end{aligned}
$$

$$
\begin{aligned}
T\left(r, \psi_{a}\right) \leqq & m\left(r, G_{a}\right)+m\left(r, H_{a}\right)+3\left\{\bar{N}\left(r, 0, W_{a}\right)+\bar{N}(r, g)\right\} \\
& +O\left\{\log ^{+} T\left(r, H_{a}\right)+\log r\right\}
\end{aligned}
$$

as $r \rightarrow \infty$, n.e.. Also from (4.3), (4.4), (4.5) and (4.6),

and

$$
T\left(r, G_{a}\right) \leqq m\left(r, W_{a}{ }^{\prime} / W_{a}\right)+\bar{N}\left(r, 0, W_{a}\right)+\bar{N}(r, g),
$$

$$
\begin{aligned}
T\left(r, H_{a}\right) \leqq & 2 m\left(r, g^{\prime} / g\right)+m\left(r, W_{a}^{\prime} / W_{a}\right)+2\left\{\bar{N}\left(r, 0, W_{a}\right)+\bar{N}(r, g)\right\} \\
& +O\left\{\log ^{+} T\left(r, g^{\prime} / g\right)+\log r\right\}
\end{aligned}
$$


as $r \rightarrow \infty$, n.e.. Combining them with the former two, we obtain the following two estimates:

$$
\begin{aligned}
T\left(r, \phi_{a}\right) \leqq & 2 m\left(r, \frac{g^{\prime}}{g}\right)+3 m\left(r, \frac{W_{a}^{\prime}}{W_{a}}\right)+2\left\{\bar{N}\left(r, 0, W_{a}\right)+\bar{N}(r, g)\right\} \\
& +O\left[\log ^{+} T\left(r, \frac{g^{\prime}}{g}\right)+\log ^{+} m\left(r, \frac{W_{a}^{\prime}}{W_{a}}\right)\right. \\
& \left.+\log ^{+}\left\{\bar{N}\left(r, 0, W_{a}\right)+\bar{N}(r, g)\right\}+\log r\right]
\end{aligned}
$$

and

$$
\begin{aligned}
T\left(r, \psi_{a}\right) \leqq & 2 m\left(r, \frac{g^{\prime}}{g}\right)+2 m\left(r, \frac{W_{a}^{\prime}}{W_{a}}\right)+3\left\{\bar{N}\left(r, 0, W_{a}\right)+\bar{N}(r, g)\right\} \\
& +O\left[\log ^{+} T\left(r, \frac{g^{\prime}}{g}\right)+\log ^{+} m\left(r, \frac{W_{a}^{\prime}}{W_{a}}\right)\right. \\
& \left.+\log ^{+}\left\{\bar{N}\left(r, 0, W_{a}\right)+\bar{N}(r, g)\right\}+\log r\right]
\end{aligned}
$$

as $r \rightarrow \infty$, n.e.. By virtue of (4.1) the characteristic function of $g^{\prime} / g$ is now given by

$$
T\left(r, \frac{g^{\prime}}{g}\right) \leqq T\left(r, \phi_{a}\right)+T\left(r, \phi_{a}\right)+O(1) .
$$

Hence from (4.11) and (4.12) we conclude that

$$
\begin{aligned}
T\left(r, \frac{g^{\prime}}{g}\right) \leqq & 4 m\left(r, \frac{g^{\prime}}{g}\right)+5 m\left(r, \frac{W_{a}^{\prime}}{W_{a}}\right)+5\left\{\bar{N}\left(r, 0, W_{a}\right)+\bar{N}(r, g)\right\} \\
& +O\left[\log ^{+} T\left(r, \frac{g^{\prime}}{g}\right)+\log ^{+} m\left(r, \frac{W_{a}^{\prime}}{W_{a}}\right)\right. \\
& \left.+\log ^{+}\left\{\bar{N}\left(r, 0, W_{a}\right)+\bar{N}(r, g)\right\}+\log r\right]
\end{aligned}
$$

as $r \rightarrow \infty$, n.e., which is the inequality as claimed.

Subcase ii. $a=0$.

Then (3.8) becomes $\phi_{0}=-2 H_{0}$. Refining (4.4) in this case we have

$$
\begin{aligned}
m\left(r, H_{0}\right) & =m\left(r, \frac{g^{\prime}}{g}\left\{\frac{\left(g^{\prime} / g\right)^{\prime}}{\left(g^{\prime} / g\right)}+\frac{g^{\prime}}{g}\right\}\right) \\
& \leqq 2 m\left(r, \frac{g^{\prime}}{g}\right)+O\left\{\log ^{+} T\left(r, \frac{g^{\prime}}{g}\right)+\log r\right\}
\end{aligned}
$$

as $r \rightarrow \infty$, n. e.. Therefore this together with (4.6) leads to an estimate

$$
T\left(r, \phi_{0}\right) \leqq 2 m\left(r, \frac{g^{\prime}}{g}\right)+2\left\{\bar{N}\left(r, 0, W_{0}\right)+\bar{N}(r, g)\right\}+O\left\{\log ^{+} T\left(r, \frac{g^{\prime}}{g}\right)+\log r\right\}
$$


as $r \rightarrow \infty$, n. e.. On the other hand (3.9) becomes $\phi_{0}=H_{0}{ }^{\prime}+G_{0} H_{0}$. It gives

$$
\begin{aligned}
m\left(r, \phi_{0}\right) \leqq & m\left(r, H_{0}\right)+m\left(r, G_{0}\right)+m\left(r, \frac{H_{0}{ }^{\prime}}{H_{0}}\right)+O(1) \\
\leqq & 2 m\left(r, \frac{g^{\prime}}{g}\right)+m\left(r, \frac{W_{0}^{\prime}}{W_{0}}\right)+O\left[\log ^{+} T\left(r, \frac{g^{\prime}}{g}\right)\right. \\
& \left.+\log ^{+}\left\{\bar{N}\left(r, 0, W_{0}\right)+\bar{N}(r, g)\right\}+\log r\right],
\end{aligned}
$$

as $r \rightarrow \infty$, n.e. by (4.3) and (4.13), because of $H_{0} \not \equiv 0$. Estimation of (4.10) is now valid as well, so we have

$$
\begin{aligned}
T\left(r, \phi_{0}\right) \leqq & 2 m\left(r, \frac{g^{\prime}}{g}\right)+m\left(r, \frac{W_{0}^{\prime}}{W_{0}}\right)+3\left\{\bar{N}\left(r, 0, W_{0}\right)+\bar{N}(r, g)\right\} \\
& +O\left[\log ^{+} T\left(r, \frac{g^{\prime}}{g}\right)+\log ^{+}\left\{\bar{N}\left(r, 0, W_{0}\right)+\bar{N}(r, g)\right\}+\log r\right]
\end{aligned}
$$

as $r \rightarrow \infty$, n. e.. Hence we can estimate the logarithmic derivative in terms of $W_{0}$ by an inequality

$$
\begin{aligned}
T\left(r, \frac{g^{\prime}}{g}\right) \leqq & 4 m\left(r, \frac{g^{\prime}}{g}\right)+m\left(r, \frac{W_{0}^{\prime}}{W_{0}}\right)+5\left\{\bar{N}\left(r, 0, W_{0}\right)+\bar{N}(r, g)\right\} \\
& +O\left[\log ^{+} T\left(r, \frac{g^{\prime}}{g}\right)+\log ^{+}\left\{\bar{N}\left(r, 0, W_{0}\right)+\bar{N}(r, g)\right\}+\log r\right]
\end{aligned}
$$

as $r \rightarrow \infty$, n. e..

Subcase iii. $a=1 / 2$.

Then (3.8) becomes $\phi_{1 / 2}=-(1 / 4) G_{1 / 2}{ }^{2}$. Since we have

$$
m\left(r, \phi_{1 / 2}\right) \leqq 2 m\left(r, G_{1 / 2}\right)=2 m\left(r, \frac{W_{1 / 2}^{\prime}}{W_{1 / 2}}\right)
$$

and

$$
N\left(r, \phi_{1 / 2}\right)=2 N\left(r, G_{1 / 2}\right) \leqq 2\left\{\bar{N}\left(r, 0, W_{1 / 2}\right)+\bar{N}(r, g)\right\}
$$

by (4.3) and (4.5), it follows

$$
T\left(r, \phi_{1 / 2}\right) \leqq 2 m\left(r, \frac{W_{1 / 2}{ }^{\prime}}{W_{1 / 2}}\right)+2\left\{\bar{N}\left(r, 0, W_{1 / 2}\right)+\bar{N}(r, g)\right\} .
$$

Similarly (3.9) becomes $\phi_{1 / 2}=(1 / 2) G_{1 / 2} H_{1 / 2}$. Reconsidering (4.4) as $a=1 / 2$ we refine it by

$$
\begin{aligned}
m\left(r, H_{1 / 2}\right) & \leqq m\left(r, \frac{g^{\prime}}{g}\right)+m\left(r, G_{1 / 2}\right) \\
& =m\left(r, \frac{g^{\prime}}{g}\right)+m\left(r, \frac{W_{1 / 2}^{\prime}}{W_{1 / 2}}\right)
\end{aligned}
$$


By this and (4.3),

$$
\begin{aligned}
m\left(r, \phi_{1 / 2}\right) & \leqq m\left(r, G_{1 / 2}\right)+m\left(r, H_{1 / 2}\right) \\
& \leqq m\left(r, \frac{g^{\prime}}{g}\right)+2 m\left(r, \frac{W_{1 / 2}^{\prime}}{W_{1 / 2}}\right),
\end{aligned}
$$

which together with (4.10) gives

$$
T\left(r, \phi_{1 / 2}\right) \leqq m\left(r, \frac{g^{\prime}}{g}\right)+2 m\left(r, \frac{W_{1 / 2}^{\prime}}{W_{1 / 2}}\right)+3\left\{\bar{N}\left(r, 0, W_{1 / 2}\right)+\bar{N}(r, g)\right\}
$$

Hence we are led to

$$
\begin{aligned}
T\left(r, \frac{g^{\prime}}{g}\right) & \leqq T\left(r, \phi_{1 / 2}\right)+T\left(r, \phi_{1 / 2}\right)+O(1) \\
& \leqq m\left(r, \frac{g^{\prime}}{g}\right)+4 m\left(r, \frac{W_{1 / 2}^{\prime}}{W_{1 / 2}}\right)+5\left\{\bar{N}\left(r, 0, W_{1 / 2}\right)+\bar{N}(r, g)\right\}+O(1) .
\end{aligned}
$$

Subcase iv. $a=1$.

In this case Estimates (3.8) and (3.9) become $\phi_{1}=G_{1}{ }^{\prime}$ and $\phi_{1}=-H_{1}{ }^{\prime}$, respectively. Because of $G_{1}{ }^{\prime} \neq 0$ and $H_{1}{ }^{\prime} \neq 0$ we deduce that

$$
\begin{aligned}
& m\left(r, \phi_{1}\right) \leqq m\left(r, G_{1}\right)+O\left[\log ^{+} T\left(r, G_{1}\right)+\log r\right], \\
& m\left(r, \phi_{1}\right) \leqq m\left(r, H_{1}\right)+O\left[\log ^{+} T\left(r, H_{1}\right)+\log r\right],
\end{aligned}
$$

as $r \rightarrow \infty$, n. e., and

$$
\begin{aligned}
& N\left(r, \phi_{1}\right)=N\left(r, G_{1}\right)+\bar{N}\left(r, G_{1}\right)=2 \bar{N}\left(r, G_{1}\right), \\
& N\left(r, \phi_{1}\right)=N\left(r, H_{1}\right)+\bar{N}\left(r, H_{1}\right)=3 \bar{N}\left(r, H_{1}\right) .
\end{aligned}
$$

Using (4.3), (4.4), (4.5) and (4.6) we get

$$
\begin{aligned}
m\left(r, \phi_{1}\right) \leqq & m\left(r, \frac{W_{1}^{\prime}}{W_{1}}\right)+O\left[\log ^{+} m\left(r, \frac{W_{1}^{\prime}}{W_{1}}\right)+\log ^{+}\left\{\bar{N}\left(r, 0, W_{1}\right)+\bar{N}(r, g)\right\}+\log r\right], \\
m\left(r, \phi_{1}\right) \leqq & 2 m\left(r, \frac{g^{\prime}}{g}\right)+m\left(r, \frac{W_{1}^{\prime}}{W_{1}}\right) \\
& +O\left[\log ^{+} T\left(r, \frac{g^{\prime}}{g}\right)+\log ^{+} m\left(r, \frac{W_{1}^{\prime}}{W_{1}}\right)\right. \\
& \left.+\log ^{+}\left\{\bar{N}\left(r, 0, W_{1}\right)+\bar{N}(r, g)\right\}+\log r\right]
\end{aligned}
$$

as $r \rightarrow \infty$, n. e., and

$$
\begin{aligned}
& N\left(r, \phi_{1}\right) \leqq 2\left\{\bar{N}\left(r, 0, W_{1}\right)+\bar{N}(r, g)\right\} \\
& N\left(r, \phi_{1}\right) \leqq 3\left\{\bar{N}\left(r, 0, W_{1}\right)+\bar{N}(r, g)\right\}
\end{aligned}
$$


Then our desired estimate is of an inequality

$$
\begin{aligned}
T\left(r, \frac{g^{\prime}}{g}\right) \leqq & 2 m\left(r, \frac{g^{\prime}}{g}\right)+2 m\left(r, \frac{W_{1}^{\prime}}{W_{1}}\right)+5\left\{\bar{N}\left(r, 0, W_{1}\right)+\bar{N}(r, g)\right\} \\
& +O\left[\log ^{+} T\left(r, \frac{g^{\prime}}{g}\right)+\log ^{+} m\left(r, \frac{W_{1}^{\prime}}{W_{1}}\right)+\log ^{+}\left\{\bar{N}\left(r, 0, W_{1}\right)+\bar{N}(r, g)\right\}+\log r\right],
\end{aligned}
$$

as $r \rightarrow \infty$, n. e.. ber $a$.

Hence in Case I the inequality (2.1) never fails to hold for any fixed num-

\section{Proof of Theorem: Case II where $\phi_{a} \equiv 0$}

Since $g \neq 0,(3.10)$ is reduced to $\phi_{a} \equiv 0$. Therefore the following two equations are given;

$$
\begin{aligned}
& a(2 a-1) G_{a}{ }^{\prime}(z)+a(a-1) G_{a}(z)^{2}-2(a-1)(2 a-1) H_{a}(z) \equiv 0, \\
& (2 a-1) H_{a}{ }^{\prime}(z)+(a-1) G_{a}(z) H_{a}(z) \equiv 0 .
\end{aligned}
$$

We now distinguish the cases with respect to the value of $a$ and determine all the forms of $g(z)$ to satisfy the two equations above.

Subcase i. $a=0$.

Then these become the equations $H_{0}(z) \equiv 0$ and $H_{0}{ }^{\prime}(z)+G_{0}(z) H_{0}(z) \equiv 0$, which we can reduce to $H_{0}(z) \equiv 0$. Applying this to $(3.5)$ we see that $g^{\prime \prime}(z) \equiv 0$. This is however the $g(z)$ listed in $\S 1,3^{\circ}$, so that $W_{0}(z) \equiv 0$. Hence $\phi_{0}$ cannot vanish identically if $a=0$.

Subcase ii. $a=1 / 2$.

Then Equations (5.1) and (5.2) become $G_{1 / 2}(z)^{2} \equiv 0$ and $G_{1 / 2}(z) H_{1 / 2}(z) \equiv 0$. Using Equation (3.5) with $a=1 / 2$ and $G_{1 / 2}(z) \equiv 0$ we get $H_{1 / 2}(z) \equiv 0$ by $g \not 0$. Applying these to (3.4) we see that $g^{\prime \prime \prime}(z) \equiv 0$, so that

$$
g(z)=\alpha z^{2}+\beta z+\gamma, \quad \text { where } \alpha, \beta, \gamma \equiv \boldsymbol{C} .
$$

For this $g(z)$ we find

$$
W_{1 / 2}(z)=W\left(\frac{1}{2} \beta z+\gamma, 2 \alpha z+\beta\right) \equiv-\frac{1}{2}\left(\beta^{2}-4 \alpha \gamma\right) .
$$

Further the constants $\alpha, \beta, \gamma$ must be taken as $\beta^{2}-4 \alpha \gamma \neq 0$ in (1) (and then clearly $G_{1 / 2}=H_{1 / 2} \equiv 0$ ). It is such a condition that immediately follows from the negation of that in $\S 1,3^{\circ}$ with $m=2$ as well.

Subcase iii. $a=1$.

Then Equations (5.1) and (5.2) become $G_{1}{ }^{\prime}(z) \equiv 0$ and $H_{1}{ }^{\prime}(z) \equiv 0$. Therefore 
we can obtain the functions $g(z)$ to be determined as entire solutions of a second order linear differential equation

$$
w^{\prime \prime}+k_{1} w^{\prime}+k_{0} w=0
$$

with the constant coefficients $k_{1}$ and $k_{0}$. Let $\lambda_{1}$ and $\lambda_{2}$ be the roots of its characteristic equation $\lambda^{2}+k_{1} \lambda+k_{0}=0$. If $\lambda_{1} \neq \lambda_{2}$, a general solution of this (5.3) is given by

$$
w=C_{1} e^{\lambda_{1} z}+C_{2} e^{\lambda_{2} z}
$$

for arbitrary constants $C_{1}, C_{2}$. In order that $W_{1}(z)=W\left(w, w^{\prime}\right)=C_{1} C_{2}\left(\lambda_{1}-\lambda_{2}\right)^{2}$. $\exp \left\{\left(\lambda_{1}+\lambda_{2}\right) z\right\}$ does not vanish identically, both $C_{1}$ and $C_{2}$ should differ from zero. If $\lambda_{1}=\lambda_{2}=\lambda$, say, a general solution to (5.3) has a form

$$
w=\left(C_{2} z+C_{1}\right) e^{\lambda z},
$$

where $C_{1}$ and $C_{2}$ are arbitrary constants. For the $w$ we have $W\left(w, w^{\prime}\right)=$ $-C_{2}{ }^{2} \exp (2 \lambda z)$. Hence it is sufficient for our purpose to choose a non-zero constant $C_{2}$ in (3). In this subcase $g(z)$ must be of the form either (2) or (3) for suitable constants $C_{1}$ and $C_{2}$.

Subcase iv. $a$ is different from $0,1 / 2,1$.

Firstly suppose that $H_{a}(z) \equiv 0$. Then Equation (3.5) gives

$$
(2 a-1) g^{\prime \prime}(z)=-a g^{\prime}(z) G_{a}(z)
$$

and (5.1) also gives

$$
(2 a-1) G_{a}{ }^{\prime}(z)+(a-1) G_{a}(z)^{2}=0 .
$$

If $G_{a}(z) \equiv 0, g^{\prime \prime}(z) \equiv 0$ by $(5.4)$, so that

$$
g(z)=\alpha z+\beta
$$

for $\alpha(\neq 0), \beta, \subseteq C$. Then $W_{a}(z) \equiv-a \alpha^{2} \neq=0$. Unless $G_{a} \equiv 0$, Equation (5.5) leads us to

$$
G_{a}(z)=\frac{2 a-1}{a-1} \cdot \frac{1}{z-z_{0}}, \quad z_{0} \in C,
$$

and then Equation (5.4) gives

$$
\frac{g^{\prime \prime}(z)}{g^{\prime}(z)}=-\frac{a}{2 a-1} G_{a}(z)=-\frac{a}{a-1} \cdot \frac{1}{z-z_{0}} .
$$

Therefore it follows

$$
g^{\prime}(z)=C\left(z-z_{0}\right)^{-a /(a-1)}, \quad C \in C-\{0\},
$$

so that $-a /(a-1)=m$, say, is an integer different from $0,-1,1$, and 


$$
g(z)=\frac{C}{m+1}\left\{\left(z-z_{0}\right)^{m+1}-\zeta\right\}, \quad \zeta \in C .
$$

Noting $a=m /(m+1)$ we see that $\zeta \neq 0$ in order that $W_{a}(z)=-a C^{2} \zeta\left(z-z_{0}\right)^{m-1}$ should not vanish identically.

After this we may suppose that $H_{a}(z) \not \equiv 0$. By Equation (5.2) we obtain

$$
G_{a}(z)=-\frac{2 a-1}{a-1} \cdot \frac{H_{a}{ }^{\prime}(z)}{H_{a}(z)} .
$$

Then (5.1) gives

$$
\begin{aligned}
2(a-1)(2 a-1) H_{a}(z) & =-\frac{a(2 a-1)^{2}}{a-1}\left(\frac{H_{a}{ }^{\prime}(z)}{H_{a}(z)}\right)^{\prime}+\frac{a(2 a-1)^{2}}{a-1}\left(\frac{H_{a}{ }^{\prime}(z)}{H_{a}(z)}\right)^{2} \\
& =-\frac{a(2 a-1)^{2}}{a-1} \cdot \frac{H_{a}{ }^{\prime \prime}(z) H_{a}(z)-2 H_{a}{ }^{\prime}(z)^{2}}{H_{a}(z)^{2}},
\end{aligned}
$$

and thus

$$
\left(\frac{1}{H_{a}(z)}\right)^{\prime \prime}=-\frac{H_{a}^{\prime \prime}(z) H_{a}(z)-2 H_{a}^{\prime}(z)^{2}}{H_{a}(z)^{3}} \equiv \frac{2(a-1)^{2}}{a(2 a-1)} .
$$

Hence we deduce

$$
H_{a}(z)=\frac{a(2 a-1)}{(a-1)^{2}} \cdot \frac{1}{(z-\alpha)(z-\beta)}, \quad \alpha, \beta \in C
$$

In virtue of this expression we get

$$
G_{a}(z)=\frac{2 a-1}{a-1}\left\{\frac{1}{z-\alpha}+\frac{1}{z-\beta}\right\}
$$

from Equation (5.6) and thus it follows from (3.2) that

$$
W_{a}(z)=C\{(z-\alpha)(z-\beta)\}^{-(2 a-1) /(a-1)}
$$

for a non-zero constant $C$. Here $-(2 a-1) /(a-1)=m$, say, is a number different from $-2,-1$, and 0 , which is equal to an integer if $\alpha \neq \beta$ and to half an integer if $\alpha=\beta$. Then we can transform (3.5) into an equation

$$
g^{\prime \prime}(z)-(m+1)\left(\frac{1}{z-\alpha}+\frac{1}{z-\beta}\right) g^{\prime}(z)+\frac{(m+1)(m+2)}{(z-\alpha)(z-\beta)} g(z)=0 .
$$

Therefore $g(z)$ is able to posess the poles possibly at $z=\alpha$ or $\beta$. Let $f(z)$ be an entire function with $f(\alpha) \neq 0$ and $f(\beta) \neq 0$, and both $k$ and $l$ be integers if $\alpha \neq \beta$ and half integers with $k=l$ if $\alpha=\beta$, such that

$$
g(z)=(z-\alpha)^{k}(z-\beta)^{l} f(z) .
$$

Using Equation (5.8) we write 


$$
\frac{g^{\prime \prime}(z)}{g(z)}=(m+1)\left(\frac{1}{z-\alpha}+\frac{1}{z-\beta}\right) \frac{g^{\prime}(z)}{g(z)}-\frac{(m+1)(m+2)}{(z-\alpha)(z-\beta)}
$$

and thus an expression

$$
\begin{aligned}
\frac{W_{a}(z)}{g(z)^{2}} & =\frac{g^{\prime \prime}(z)}{g(z)}-\frac{m+1}{m+2}\left(\frac{g^{\prime}(z)}{g(z)}\right)^{2} \\
& =-\frac{m+1}{m+2}\left\{\left(\frac{g^{\prime}(z)}{g(z)}\right)^{2}-(m+2)\left(\frac{1}{z-\alpha}+\frac{1}{z-\beta}\right) \frac{g^{\prime}(z)}{g(z)}+\frac{(m+2)^{2}}{(z-\alpha)(z-\beta)}\right\} \\
& =-\frac{m+1}{m+2}\left(\frac{g^{\prime}(z)}{g(z)}-\frac{m+2}{z-\alpha}\right)\left(\frac{g^{\prime}(z)}{g(z)}-\frac{m+2}{z-\beta}\right) \\
& =-\frac{m+1}{m+2}\left(\frac{k-m-2}{z-\alpha}+\frac{l}{z-\beta}+\frac{f^{\prime}(z)}{f(z)}\right)\left(\frac{k}{z-\alpha}+\frac{l-m-2}{z-\beta}+\frac{f^{\prime}(z)}{f(z)}\right) .
\end{aligned}
$$

On the other hand, (5.7) gives

$$
\frac{W_{a}(z)}{g(z)^{2}}=\frac{C}{(z-\alpha)^{2 k-m}(z-\beta)^{2 l-m} f(z)^{2}} .
$$

In the case where $\alpha \neq \beta, g(z)$ is expressed by

$$
g(z)=c_{\alpha}(z-\alpha)^{k}\{1+O(z-\alpha)\}, \quad c_{\alpha} \subseteq C-\{0\}
$$

in a neighborhood of $z=\alpha$. Substituting this into the equation (5.8) and comparing the coefficients of the term $(z-\alpha)^{k-2}$, we get a characteristic equation $k(k-m-2)=0$, so that $k$ can be of the value 0 or $m+2$. Then it immediately follows that $m=-3$ and $k=-1$, when we compare the behavior of two expressions above for $W_{a}(z) / g(z)^{2}$ in a neighborhood of $z=\alpha$. In fact we see that $2 k-m=1$ in both cases of $k=0$ and $k=m+2$. We have $m=-3$ when $k=m+2$, while $m=-1$ when $k=0$. The latter is now excluded. The same is true of the number $l$. Hence $a=2$ and

$$
g(z)=\frac{f(z)}{(z-\alpha)(z-\beta)}
$$

if $\alpha \neq \beta$. Concerning $f(z)$ we have

$$
\frac{C}{(z-\alpha)(z-\beta) f(z)^{2}}=-2\left\{-\frac{1}{z-\beta}+\frac{f^{\prime}(z)}{f(z)}\right\}\left\{-\frac{1}{z-\alpha}+\frac{f^{\prime}(z)}{f(z)}\right\}
$$

and thus

$$
\left\{(z-\beta) f^{\prime}(z)-f(z)\right\}\left\{(z-\alpha) f^{\prime}(z)-f(z)\right\} \equiv-\frac{C}{2} \quad(\neq 0) .
$$

Differentiating both sides of this, we get an identity

$$
(z-\beta) f^{\prime \prime}(z)\left\{(z-\alpha) f^{\prime}(z)-f(z)\right\}=-(z-\alpha) f^{\prime \prime}(z)\left\{(z-\beta) f^{\prime}(z)-f(z)\right\} .
$$

Unless $f^{\prime \prime}(z) \equiv 0$, 


$$
(z-\beta)\left\{(z-\alpha) f^{\prime}(z)-f(z)\right\}=-(z-\alpha)\left\{(z-\beta) f^{\prime}(z)-f(z)\right\}
$$

and therefore

$$
(\alpha-\beta) f(\alpha)=0 \text {. }
$$

This is impossible, so that $f^{\prime \prime}(z) \equiv 0$, i. e., $f(z)=D(z-\gamma)$ where $D \cong \boldsymbol{C}-\{0\}$ and $\gamma \subseteq C-\{\alpha, \beta\}$. Then

$$
g(z)=\frac{D(z-\gamma)}{(z-\alpha)(z-\beta)},
$$

which satisfies the condition (5.7) with $C=-2 D^{2}(\gamma-\alpha)(\gamma-\beta)$. to

Next we shall consider the case where $\alpha=\beta$. Equation (5.8) is then equal

$$
g^{\prime \prime}(z)-\frac{2(m+1)}{z-\alpha} g^{\prime}(z)+\frac{(m+1)(m+2)}{(z-\alpha)^{2}} g(z)=0
$$

Here we make a similar discussion to the above with

$$
g(z)=(z-\alpha)^{2 k} f(z),
$$

and obtain a characteristic equation

$$
2 k(2 k-1)-2 k \cdot 2(m+1)+(m+1)(m+2)=0,
$$

and so $2 k=m+1$ or $2 k=m+2$. Since we now have

$$
\frac{W_{a}(z)}{g(z)^{2}}=\frac{C}{(z-\alpha)^{2(2 k-m)} f(z)^{2}}=-\frac{m+1}{m+2}\left\{\frac{2 k-m-2}{z-\alpha}+\frac{f^{\prime}(z)}{f(z)}\right\}^{2},
$$

the latter, $2 k=m+2$, gives immediately a contradiction as $2 k-m: \neq 0$. For the former case where $2 k=m+1$ the behavior of two expressions above for $W_{a}(z) / g(z)^{2}$ is compatible. Then $f(z)$ satisfies the relation

$$
\left\{(z-\alpha) f^{\prime}(z)-f(z)\right\}^{2} \equiv-\frac{m+2}{m+1} \cdot C(\neq 0) .
$$

Differentiatıng this we have $f^{\prime \prime}(z) \equiv 0$, or $f(z)=D(z-\gamma), D \equiv \boldsymbol{C}-\{0\}, \gamma \boxminus \boldsymbol{C}-\{\alpha\}$ again. Hence if $\alpha=\beta, a=(m+1) /(m+2)$ and

$$
g(z)=D(z-\alpha)^{m+1}(z-\gamma),
$$

provided that $m$ is an integer different from $0,-1$, and -2 . In order that $g(z)$ may satisfy (5.7), i. e.,

$$
W_{a}(z)=C(z-\alpha)^{2 m},
$$

we choose the constant $C=-a D^{2}(\gamma-\alpha)^{2}$.

We have discussed all the possible forms that $g(z)$ has in Case II :

(1) when $a=1 / 2, g(z)=\alpha z^{2}+\beta z+\gamma$, where $\beta^{2}-4 \alpha \gamma \neq 0$; 
(2) when $a=1, g(z)=C_{1} e^{\lambda_{1} z}+C_{2} e^{\lambda_{2} z}$, where $\lambda_{1} \pm \lambda_{2}$ and $C_{1} C_{2} \neq 0$;

(3) when $a=1, g(z)=\left(C_{2} z+C_{1}\right) e^{\lambda z}$, where $C_{2} \neq 0$;

(4) when $a \neq 0,1 / 2,1, g(z)=C_{1}(z-\alpha)$, where $C_{1} \neq 0$;

(5) when $a=(m-1) / m, g(z)=C_{2}\left\{(z-\alpha)^{m}-C_{1}\right\}$, where $C_{1} C_{2} \neq 0$ and $m \neq 0$, 1,2 ;

(6) when $a=2, g(z)=\left(C_{1}(z-\gamma) /(z-\alpha)(z-\beta)\right)$, where $C_{1} \neq 0$, and $\alpha, \beta, \gamma$ are mutually distinct;

(7) when $a=(m+1) /(m+2), g(z)=C_{1}(z-\alpha)^{m+1}(z-\gamma)$, where $C_{1} \neq 0, \alpha \neq \gamma$ and $m \neq 0,-1,-2$,

provided that $C_{1}, C_{2}, \lambda_{1}, \lambda_{2}, \lambda, \alpha, \beta, \gamma \equiv C$ and $m$ is an integer. As their $W_{a}(z)$ we obtain also

(1) $W_{1 / 2}(z) \equiv-(1 / 2)\left(\beta^{2}-4 \alpha \gamma\right)$;

(2) $W_{1}(z)=C_{1} C_{2}\left(\lambda_{1}-\lambda_{2}\right)^{2} e^{\left(\lambda_{1}+\lambda_{2}\right) z}$;

(3) $W_{1}(z)=-C_{2}^{2} e^{2 \lambda z}$;

(4) $W_{a}(z) \equiv-a \alpha^{2}$;

(5) $W_{(m-1) / m}(z)=-m(m-1) C_{1} C_{2}(z-\alpha)^{m-2}$;

(6) $W_{2}(z)=\left(-2 C_{1}^{2}(\gamma-\alpha)(\gamma-\beta)\right) /\left((z-\alpha)^{3}(z-\beta)^{3}\right)$;

(7) $W_{(m+1) /(m+2)}(z)=-\{(m+1) /(m+2)\} C_{1}{ }^{2}(\gamma-\alpha)^{2}(z-\alpha)^{2 m}$.

Finally we need to examine whether the inequality (2.1) holds or not in each case above. The function $U_{a}(r)$ in (2.1) grows at least as rapidly as $O(\log r)$ for $r \rightarrow \infty$, n.e.. Therefore (2.1) is satisfied by $g(z)$ given in (4), (5), (6) and (7) as rational functions. As proved in Remark $2^{\circ}$ in $\S 2$, two possibilities (1) and (2) are the very exceptions. With $g(z)$ as in (3) it is easily shown that

and

$$
\begin{aligned}
& m\left(r, g^{\prime} / g\right)=O(1), \quad m\left(r, W_{1}^{\prime} / W_{1}\right)=O(1), \\
& \bar{N}\left(r, 0, W_{1}\right)=\bar{N}(r, g) \equiv 0,
\end{aligned}
$$

$$
T\left(r, g^{\prime} / g\right)=\log r+O(1),
$$

as $r \rightarrow \infty$. Then $U_{a}(r)=O(\log r)$ as $r$ tends to infinity, so Inequality (2.1) also holds. This completes the proof of the theorem.

Remark. Mention needs to be made of rational functions. Reconsidering the above proof in Case I as a rational function $g(z)$, we see that all of $m\left(r, G_{a}\right)$, $m\left(r, H_{a}\right), m\left(r, \phi_{a}\right)$ and $m\left(r, \phi_{a}\right)$ grow possibly in the degree of $o(1)$ with the aid of (1.2) in Lemma. Inequality (2.1) can be therefore sharpened by

$$
T\left(r, \frac{g^{\prime}}{g}\right) \leqq 5\left\{\bar{N}\left(r, 0, W_{a}\right)+\bar{N}(r, g)\right\}+O(1) .
$$

Then there exist such the rational functions $g(z)$ as never satisfy (5.9) only in (3) with $\lambda=0$, (4), (5) with $m>5$ or $m<-4$, as well as (1) of Case II. In fact, since Inequality (5.9) equals 


$$
\bar{N}(r, 0, g) \leqq 5 \bar{N}\left(r, 0, W_{a}\right)+4 \bar{N}(r, g)+O(1)
$$

in virtue of the equation

$$
T\left(r, \frac{g^{\prime}}{g}\right)=m\left(r, \frac{g^{\prime}}{g}\right)+N\left(r, \frac{g^{\prime}}{g}\right)=\bar{N}(r, 0, g)+\bar{N}(r, g)+o(1),
$$

this fact can be shown by studying these counting functions in each occasion. In (5) for example, if $m>2$,

$$
\bar{N}(r, 0, g)=m \log r, \quad \bar{N}\left(r, 0, W_{a}\right)=\log r, \quad \bar{N}(r, g) \equiv 0
$$

and if $m<0$,

$$
\bar{N}(r, 0, g)=-m \log r, \quad \bar{N}\left(r, 0, W_{a}\right) \equiv 0, \quad \bar{N}(r, g)=\log r
$$

for sufficiently large $r$. The equality of (5.9) occurs if $m=5$ or $m=-4$.

\section{Proof of Corollary}

In order to prove this result we shall return to the proof of Theorem. At first we are concerned about Case I in Section 4. Assume that $\varphi_{a}(z) \neq 0$. The present assumption (2.2) reduces the equations (4.3) and (4.4) to

and

$$
m\left(r, G_{a}\right)=m\left(r, \frac{W_{a}^{\prime}}{W_{a}}\right)=S\left(r, W_{a}\right)=S(r, g)
$$

$$
m\left(r, H_{a}\right)=S(r, g)+S\left(r, W_{a}\right)=S(r, g),
$$

respectively. Similarly (4.5) and (4.6) become

$$
N\left(r, G_{a}\right) \leqq \bar{N}\left(r, 0, W_{a}\right)=m\left(r, W_{a}\right)+O(1)=S(r, g)
$$

and

$$
N\left(r, H_{a}\right) \leqq 2 \bar{N}\left(r, 0, W_{a}\right)=S(r, g) .
$$

All of them hold independently of the value of $a \in C$. Therefore it follows also for both $\phi_{a}$ and $\phi_{a}$ that

$$
T\left(r, \phi_{a}\right)=S(r, g) \text { and } T\left(r, \phi_{a}\right)=S(r, g),
$$

so that

$$
T\left(r, \frac{g^{\prime}}{g}\right)=S(r, g)
$$

Using a relation

$$
g^{2}=\frac{W_{a}}{\left(\frac{g^{\prime}}{g}\right)^{\prime}-(a-1)\left(\frac{g^{\prime}}{g}\right)^{2}},
$$


we obtain

$$
\begin{aligned}
2 T(r, g) & \leqq T\left(r, W_{a}\right)+T\left(r,\left(\frac{g^{\prime}}{g}\right)^{\prime}-(a-1)\left(\frac{g^{\prime}}{g}\right)^{2}\right)+O(1) \\
& \leqq m\left(r, W_{a}\right)+4 T\left(r, \frac{g^{\prime}}{g}\right)+S\left(r, \frac{g^{\prime}}{g}\right)+O(1) .
\end{aligned}
$$

Then from (2.2) and (6.1) we conclude

$$
T(r, g)=S(r, g)
$$

which is impossible. Hence it must be hold $\varphi_{a}(z) \equiv 0$.

Concerning the possibilities in Case II we have made a list in Section 5. We shall pick out those what give entire functions $g(z)$ with the property (2.2). Evidently (5), (6) and (7) are beside our object. If $g(z)$ is a polynomial, $W_{a}(z)$ must be a constant. Possibilities (1) and (4) come under this heading. If $\lambda_{1}+\lambda_{2}$ $\neq 0$ in (2), then $W_{1}(z)=C_{1} C_{2}\left(\lambda_{1}-\lambda_{2}\right)^{2} e^{\left(\lambda_{1}+\lambda_{2}\right) z}$ is an entire function of order one and

$$
m\left(r, W_{1}\right)=\frac{\left|\lambda_{1}+\lambda_{2}\right|}{\pi} r+O(1) \quad \text { as } r \rightarrow \infty .
$$

(See Hayman [1], p. 7.) A similar observation shows

$$
m(r, g) \leqq\left(\left|\lambda_{1}\right|+\left|\lambda_{2}\right|\right) \frac{r}{\pi}+O(1) \quad \text { as } r \rightarrow \infty .
$$

Therefore (2.2) fails to hold since

$$
\lim _{r \rightarrow \infty} \frac{m\left(r, W_{1}\right)}{m(r, g)} \geqq \frac{\left|\lambda_{1}+\lambda_{2}\right|}{\left|\lambda_{1}\right|+\left|\lambda_{2}\right|}>0 .
$$

When $\lambda_{1}+\lambda_{2}=0, W_{1}(z)$ is a constant and $g(z)$ is such a transcendental entire function that satisfies all the assumptions in Corollary. Functions in the last remaining (3) can satisfy Condition (2.2) only if $\lambda=0$. We have thus proved the corollary.

\section{REFERENCES}

[1] W.K. Hayman, Meromorphic functions, Oxford University Press, 1964.

[2] W. K. HAYMAN, Research problems in function theory, University of London, The Athlone Press, 1967.

[3] W.K. HAYMAn, Picard values of meromorphic functions and their derivatives, Ann. of Math., 70 (1959), 9-42.

[4] E. Mues, Über die Nullstellen homogener Differentialpolynome, manuscripta math., 23 (1978), 325-341. 
[5] M. OzAwA, Zeros of certain differential polynomials, Analytic function theory of one complex variable (edited by) Y. Komatu, K. Niino, and C.C. Yang, Pitman research notes in mathematics series, 212 (1989), 199-225.

Department of MATHEMATICS

ScIENCE University of TOKYo

Noda, Chiba, JaPAN 